\title{
The Association Between Trimester-Specific Weight Gain and Severe Preeclampsia/Adverse Perinatal Outcome in Gestational Diabetes Mellitus Complicated by Preeclampsia: A Retrospective Case Study
}

Xueqin Zhang $\cdot$ Yunshan Xiao (D)

Received: January 14, 2019 / Published online: March 5, 2019

(C) The Author(s) 2019

\begin{abstract}
Introduction: Gestational diabetes mellitus (GDM) and preeclampsia share many risk factors, e.g., gestational weight gain (GWG). Previous studies on the co-occurrence of these two diseases cannot powerfully clear up the effects of GWG on perinatal outcome.

Methods: A total of 329 pregnant women with GDM complicated by preeclampsia were enrolled. Clinical data of mothers and newborns were retrospectively analyzed, including baseline characteristics of pregnant women and pregnancy outcomes. We focused on the association between trimester-specific weight gain and severe preeclampsia (s-PE)/adverse perinatal outcomes in GDM complicated by preeclampsia, including cesarean section (C-sect), preterm birth, and large for gestational age birth (LGA). Regression analysis was used to adjust the impact of confounding factors, including height, age, parity, scarred uterus, and so on.
\end{abstract}

Xueqin Zhang and Yunshan Xiao contributed equally to this work and should be considered co-first authors.

Enhanced Digital Features To view enhanced digital features for this article go to https://doi.org/10.6084/ m9.figshare.7726145.

X. Zhang · Y. Xiao $(\bowtie)$

Department of Obstetrics, Xiamen Maternal and Child Healthcare Hospital, Xiamen 361003, Fujian, People's Republic of China

e-mail: xyssfp@163.com
Result: By unconditional regression analysis, middle trimester excessive GWG is a risk factor for LGA [OR 6.586, 95\% CI (2.254-19.242), AOR 6.481, 95\% CI (2.213-18.981)]; late excessive GWG is a risk factor for s-PE and C-sect [OR 1.683, 95\% CI (1.084-2.614), AOR 1.888, 95\% CI (1.193-2.990); and OR 1.754, 95\% CI (1.121-2.744), AOR 1.841, 95\% CI (1.153-2.937)], excessive total GWG is a risk factor for LGA, and is a protective factor for the preterm [OR 5.920, 95\% CI (2.479-14.139), AOR 5.602, 95\% CI (2.337-13.431); and OR 0.448, 95\% CI (0.248-0.841), AOR 0.429, 95\% CI (0.235-0.783)].

Conclusions: The trimester-specific weight gain has a significant impact on the perinatal outcomes among pregnant women with both GDM and preeclampsia. This study is helpful for carry out risk monitoring in time, identifying early warning signs, and improving maternal and infant health.

Keywords: Gestational diabetes mellitus; Perinatal outcome; Preeclampsia; Trimesterspecific weight gain

\section{INTRODUCTION}

Gestational diabetes mellitus (GDM) and preeclampsia are two dangerous pregnancy complications, and their coexistence further 
increases adverse perinatal outcomes. However, studies on this situation are insufficient.

GDM is defined as glucose intolerance that occurs or is diagnosed for the first time during pregnancy without previous diagnosis of diabetes. Pregnant women with GDM have increased risks of preeclampsia, preterm birth, cesarean delivery, and secondary postpartum type 2 diabetes mellitus. Infants born to GDM women have an increased risk of macrosomia, congenital abnormalities, and secondary metabolic syndrome in the future [1].

Preeclampsia is diagnosed with new-onset hypertension and proteinuria in the second half of pregnancy, which has long-term adverse effects. The American Heart Association points out that pregnant women with preeclampsia have an increased risk of cardiovascular disease in the future [2]. Preeclampsia is classified into mild preeclampsia (m-PE) and severe preeclampsia (s-PE). s-PE is characterized by severe hypertension ( $\geq 160 / 110 \mathrm{mmHg}$ ) or endorgan injury. Women who met criteria of preeclampsia but not s-PE are diagnosed as $\mathrm{m}-\mathrm{PE}$. The threat of s-PE to mothers and infants is more serious than that of m-PE.

The development of GDM originates from insulin resistance, and that of preeclampsia is related to abnormal placentation leading to reduced placental perfusion $[1,3]$. Dyslipidemia plays a significant role in the pathogenesis of both diseases [4]. GDM and preeclampsia share similar risk factors, such as obesity related to dyslipidemia [5]. How obesity affects perinatal outcomes of GDM complicated by preeclampsia remains to be further explored.

There are two main indicators for evaluating obesity: (1) body mass index (BMI) which is calculated on the basis of body weight and height; (2) gestational weight gain (GWG). In recent years, researchers tend to analyze the time point of GWG of pregnant women with different phenotype, instead of limiting themselves to total GWG.

The indicators in this study included prepregnancy BMI, total GWG, and weight gains at the early, middle, and late pregnancy. We also use regression analysis to adjust the impact of other potential risk factors, including height, age, parity, scarred uterus, and so on. We focused on the association between trimesterspecific weight gain and s-PE/adverse perinatal outcomes in GDM complicated by preeclampsia, including preterm birth, cesarean section (C-sect), and large for gestational age birth (LGA). This study will help us to monitor the risk factors in time and improve perinatal outcomes.

\section{METHODS}

\section{Study Population and Eligibility Criteria}

We used the anonymized data of the clinical characteristics to analyze retrospectively from the electronic medical record (EMR) system of the Maternal and Child Healthcare Hospital of Xiamen City. This study was approved by the ethics committee of the Maternal and Child Healthcare Hospital of Xiamen City (ky2019-006). All procedures were in accordance with the ethical standards of the local ethics committee and with the 1964 Helsinki Declaration and its later amendments or comparable ethical standards. Informed consent was obtained from all individual participants.

The present study was a retrospective study of pregnant women who underwent prenatal examinations and delivery at the Maternal and Child Healthcare Hospital of Xiamen, China, from January 2016 to November 2018. During the period, 368 out of 70,057 (5.2\%) single pregnant women had GDM complicated by preeclampsia. According to the following criteria, 329 of them were enrolled in this study.

The inclusion criteria of the participants were as follows: (1) singleton gestation; (2) birth occurred at 28 or more gestational weeks; (3) complete mother-newborn information.

Cases meeting the following criteria were excluded: (1) multiple gestations; (2) fetuses with chromosome abnormalities or congenital malformations; (3) mothers with mental disease, alcohol or drug abuse, pregestational diabetes, and so on. 


\section{Data Collection}

We recorded the following clinical data: height, maternal age, BMI, abortion history, parity, scarred uterus, educational level, the weight of different gestational weeks, gestational weeks at delivery, mode of delivery, fetal sex, newborn birth weight, the severity of preeclampsia and perinatal outcome, including cesarean delivery, preterm birth, and LGA.

GDM diagnosis is made when any glucose values exceeded the standard cutoff levels (fasting, $5.1 \mathrm{mmol} / \mathrm{L} ; 1 \mathrm{~h}, 10.0 \mathrm{mmol} / \mathrm{L} ;$ and $2 \mathrm{~h}, 8.50 \mathrm{mmol} / \mathrm{L}$ ) in a 75 -g oral glucose tolerance test between 24 and 28 weeks of pregnancy $[6,7]$. All patients with GDM were treated with dietary adjustment or lifestyle modification combined with insulin to achieve the following blood glucose goals: FPG 3.3-5.3 mmol/l and 1-h postprandial blood glucose $<7.8 \mathrm{mmol} / \mathrm{l}$. Failure to achieve the goal is called poor glycemic control.

Preeclampsia is diagnosed by systolic blood pressure $(\geq 140 \mathrm{mmHg}$ ) and/or diastolic blood pressure $(\geq 90 \mathrm{mmHg}$ ) after 20 weeks of gestation, proteinuria ( $\geq 0.3 \mathrm{~g} / 24 \mathrm{~h}$ ) or positive random urinary protein, upper abdominal discomfort, headache, and other symptoms. $\mathrm{s}$-PE is diagnosed with any of the following adverse conditions in preeclampsia patients: Continuous elevation of blood pressure: systolic blood pressure ( $\geq 160 \mathrm{mmHg}$ ) and/or diastolic blood pressure $(\geq 110 \mathrm{mmHg})$; proteinuria $(\geq 2.0 \mathrm{~g} / 24 \mathrm{~h}$ or random proteinuria $(++)$; serum creatinine $(\geq 106 \mu \mathrm{m} / \mathrm{L})$; platelet $(<100,000 / \mathrm{ML})$; elevated blood LDH; elevated serum ALT or AST levels; persistent headache or other brain or visual impairments; and persistent upper abdominal pain [8].

The adverse perinatal outcomes include cesarean section, LGA infant (birth weights exceeded the 90th percentile for gestational age [9]), preterm birth (delivery before 37 gestational weeks).

Short stature was defined as height in the lowest quartile of adult women in China $(<155 \mathrm{~cm})$ [10]. Advanced age was defined as older than 35 years at delivery.

Pre-pregnancy weight was self-reported during the first prenatal examination. Pre- pregnancy BMI was calculated as weight (in kilograms) divided by height (in meters) squared. BMI categories were established according to the Working Group of Obesity in China (underweight, $<18.5$; normal weight, 18.5-23.9; overweight, 24-28.9; and obese, $>28.9$ ) [11]. In the present study, pre-pregnancy obesity was defined as BMI $>23.9$. Overweight and obese women were combined because the BMI of only a small number of women was more than 28.9 .

According to the US Institute of Medicine (IOM) guidelines 2009 [12], the adequate total GWG (defined as the difference between prepregnancy body weight and the last weight measurement before delivery) were underweight women (12.5-18 kg), normal weight women (11.5-16 kg), overweight women $(7-11.5 \mathrm{~kg})$, and obese women $(5-9 \mathrm{~kg})$. Early, middle, and late trimesters were defined as weeks $1-13,14-26$, and 28-40, respectively. The adequate weight gain interval is $0.5-2 \mathrm{~kg}$ for all BMI levels in early pregnancy. The adequate weight gain rates in middle and late trimester (expressed by average weekly weight gain) were underweight group (0.44-0.58 kg/week), normal weight group $(0.38-0.50 \mathrm{~kg} /$ week $)$, overweight group $(0.23-0.33 \mathrm{~kg} /$ week $)$, and obese group $(0.17-0.27 \mathrm{~kg} /$ week). Excessive GWG is defined as exceeding the upper limit of the adequate range.

\section{Statistical Analysis}

Statistical analyses were performed with the SAS software (version 9.3; SAS Institute Inc., Cary, NC). Categorical variables were described by using frequencies. The statistical difference in the prevalence of adverse perinatal outcomes was calculated using the Chi-square test.

In order to determine the odds ratio (OR) and $95 \%$ confidence interval (CI) for the association between gestational specific weight gain and perinatal outcomes of GDM with preeclampsia, and to control the influence of confounding factors, unconditional regression analysis was used. 


\section{RESULTS}

Of the 70,057 singleton pregnant women included in this study, $368(0.53 \%)$ pregnant women were diagnosed with GDM complicated by preeclampsia. In the end, 329 cases meeting the inclusion criteria were enrolled. The s-PE rate was $51.3 \%$. The preterm birth rate was $20.3 \%$. The cesarean delivery rate was $65.1 \%$. The LGA rate was $9.7 \%$. Two stillbirths were excluded from LGA analysis.

As compared to the controls, pregnant women with short stature (67.9\% vs $48.2 \%, p<$ $0.05)$, advanced age ( $63.9 \%$ vs $45.2 \%, p<0.05)$, multiparous $(59.5 \%$ vs $46.0 \%, p<0.05)$, and late excessive GWG (56.9\% vs $44.0 \%, p<0.05)$ were associated with higher s-PE rates. The preterm rate of pregnant women with scarred uterus was significantly higher than that of pregnant women without scarred uterus (35.7\% vs $18.1 \%, p<0.05)$. The preterm rate of pregnant women with excessive total GWG was significantly lower than that of women with non-late excessive GWG (13.2\% vs $25.4 \%$, $p<0.05)$. Pregnant women with scarred uterus (95.5\% vs $55.1 \%, p<0.05)$ or late excessive GWG $(66.0 \%$ vs $52.5 \%, p<0.05)$ were associated with higher $\mathrm{C}$-sect rate. The LGA rates of pregnant women with excessive total GWG $(18.4 \%$ vs $3.7 \%, p<0.05)$ or middle trimester ( $15.6 \%$ vs $2.7 \%, p<0.05$ ) excessive GWG were significantly higher than that of the controls (Tables 1 and 2).

Table 1 Prevalence of severe preeclampsia/adverse perinatal outcome in pregnant women with different clinical baseline characteristics

\begin{tabular}{|c|c|c|c|c|c|}
\hline Baseline characteristic & N1 (N2) Total 329 (327) & s-PE (\%) & Preterm (\%) & C-sect (\%) & LGA (\%) \\
\hline Short stature & $53(53)$ & $67.9^{*}$ & 20.8 & 71.7 & 3.8 \\
\hline Non-short stature ${ }^{a}$ & $276(274)$ & 48.2 & 20.3 & 58.0 & 10.9 \\
\hline Advanced age & $108(108)$ & $63.9^{*}$ & 22.2 & 65.7 & 5.6 \\
\hline Non-advanced age $\mathrm{e}^{\mathrm{a}}$ & $221(219)$ & 45.2 & 19.5 & 57.5 & 11.9 \\
\hline Multipara & $131(130)$ & $59.5^{*}$ & 25.2 & 57.3 & 10.8 \\
\hline Nulliparous $^{\mathrm{a}}$ & $198(197)$ & 46.0 & 17.2 & 62.1 & 9.1 \\
\hline Abortion history & $168(168)$ & 53.0 & 22.6 & 58.9 & 12.5 \\
\hline Non-abortion history $^{\mathrm{a}}$ & $161(159)$ & 49.7 & 18.0 & 61.5 & 6.9 \\
\hline Scarred uterus & $42(42)$ & 59.5 & $35.7^{*}$ & $95.2^{*}$ & 11.9 \\
\hline Non-scarred uterus ${ }^{a}$ & $287(285)$ & 50.2 & 18.1 & 55.1 & 9.5 \\
\hline College diploma or above & $224(224)$ & 51.3 & 21.9 & 58.0 & 8.5 \\
\hline Less than college diploma ${ }^{a}$ & $105(103)$ & 51.4 & 17.1 & 64.8 & 12.6 \\
\hline Male fetus & $177(176)$ & 53.1 & 20.3 & 59.9 & 9.7 \\
\hline Female fetus ${ }^{a}$ & $152(151)$ & 49.3 & 20.4 & 60.5 & 9.9 \\
\hline Poor glycemic control & $87(87)$ & 56.3 & 24.1 & 56.3 & 10.3 \\
\hline Non-poor glycemic control ${ }^{a}$ & $242(240)$ & 49.6 & 19.0 & 61.6 & 9.5 \\
\hline
\end{tabular}

$N 1$ number of cases analyzed for s-PE, preterm, C-sect; $N 2$ number of cases analyzed for LGA. $s-P E$ severe preeclampsia, $C$ sect cesarean section, $L G A$ large for gestational age birth

${ }^{*}$ Statistically significant compared with controls

${ }^{a}$ Control group 
Table 2 Prevalence of severe preeclampsia/adverse perinatal outcome in pregnant women with different gestational weight gain characteristics

\begin{tabular}{lllllc}
\hline Baseline characteristic & N1 (N2) Total 329 (327) & s-PE (\%) & Preterm (\%) & C-sect (\%) & LGA (\%) \\
\hline Early excessive GWG & $97(97)$ & 49.5 & 15.5 & 64.9 & 10.3 \\
Non-early excessive GWG $^{\text {a }}$ & $232(230)$ & 52.2 & 22.4 & 58.2 & 9.6 \\
Middle excessive GWG & $181(180)$ & 47.5 & 18.8 & 60.8 & $15.6^{*}$ \\
Non-mid excessive GWG $^{\text {a }}$ & $148(147)$ & 56.1 & 22.3 & 59.5 & 2.7 \\
Late excessive GWG & $188(187)$ & $56.9^{*}$ & 23.9 & $66.0^{*}$ & 11.8 \\
Non-late excessive GWG & $141(140)$ & 44.0 & 15.6 & 52.5 & 7.1 \\
Excessive total GWG & $136(136)$ & 46.3 & $13.2^{*}$ & 62.1 & $18.4^{*}$ \\
Non-excessive total GWG & $193(184)$ & 56.9 & 25.4 & 57.3 & 3.7 \\
\hline
\end{tabular}

$N 1$ number of cases analyzed for s-PE, preterm, C-sect; $N 2$ number of cases analyzed for LGA. $s-P E$ severe preeclampsia, $C$-sect cesarean section, $L G A$ large for gestational age birth

${ }^{*}$ Statistically significant compared with controls

a Control group

By unconditional regression analysis, middle trimester excessive GWG is a risk factor for LGA [OR 6.586, 95\% CI (2.254-19.242), AOR 6.481, 95\%CI (2.213-18.981)]; late excessive GWG is a risk factor for s-PE and C-sect [OR 1.683, 95\% CI (1.084-2.614), AOR 1.888, 95\% CI (1.193-2.990); and OR 1.754, 95\% CI (1.121-2.744), AOR 1.841, 95\% CI (1.153-2.937)]; excessive total GWG is a risk factor for LGA and is a protective factor for the preterm [OR 5.920, 95\% CI (2.479-14.139), AOR 5.602, 95\% CI (2.337-13.431); and OR $0.448,95 \%$ CI (0.248-0.841), AOR 0.429, 95\% CI (0.235-0.783)] (Table 3).

\section{DISCUSSION}

The incidence of GDM complicated by preeclampsia is unclear. According to the diagnostic criteria of the Ministry of Health $(\mathrm{MOH})$ in China, the prevalence of GDM is $17.5 \%$ [13], and another study reports that the prevalence of preeclampsia among patients with GDM in China is $3.3 \%$ [14]. On the basis of the above results, it is estimated that the incidence of GDM combined with preeclampsia in China is $0.57 \%$ approximately. Of the 70,057 singleton pregnant women included in this study, 368 $(0.53 \%)$ developed GDM complicated by preeclampsia. A total of 329 patients in this study were included in the analysis. The preterm birth rate was $20.3 \%$, cesarean delivery rate was $65.1 \%$, and LGA rate was $9.7 \%$. The incidence of adverse pregnancy outcomes was far higher than that of normal pregnant women $[15,16]$. GDM complicated by preeclampsia is a severe problem endangering the health of mothers and infants, and it needs special attention.

Only by thoroughly understanding the relationship between relevant risk factors, e.g., obesity and perinatal outcomes, can we monitor and take preventive measures in time. Currently, there is a lack of large-scale multicenter collaborative clinical studies on the coexistence of GDM and preeclampsia, and it is difficult to carry out meta-analyses and evidence-based medicine research with limited literature. According to the data of prenatal examinations and inpatient deliveries in a tertiary hospital for 6 years, this study retrospectively analyzed the factors influencing perinatal outcomes.

The difference in the incidence of pre-pregnancy obesity between $\mathrm{m}-\mathrm{PE}$ and s-PE is as yet unclear [17]. Pre-pregnancy obesity has been reported to increase the risk of medical-indicated preterm birth, cesarean delivery, and LGA $[18,19]$. In the present study, there was no 
Table 3 Unconditional regression analysis of the association between gestational weight gain (GWG) and severe preeclampsia/adverse perinatal outcomes

\begin{tabular}{lllll}
\hline & OR & 95\% CI & AOR & 95\% CI \\
\hline $\begin{array}{l}\text { Middle excessive GWG for LGA } \\
\text { Non-middle excessive GWG (reference) }\end{array}$ & 6.586 & $2.254-19.242$ & 6.481 & $2.213-18.981^{*}$ \\
$\begin{array}{l}\text { Late excessive GWG for s-PE } \\
\text { Non-late excessive GWG (reference) }\end{array}$ & 1.683 & $1.084-2.614$ & 1.888 & $1.193-2.990^{*}$ \\
$\begin{array}{l}\text { Late excessive GWG for C-sect } \\
\text { ( }\end{array}$ & 1.754 & $1.121-2.744$ & 1.841 & $1.153-2.937^{*}$ \\
$\begin{array}{l}\text { Non-late excessive GWG (reference) } \\
\text { Excessive total GWG for LGA }\end{array}$ & & & & \\
$\begin{array}{l}\text { Non-excessive total GWG (reference) } \\
\text { Excessive total GWG for preterm }\end{array}$ & 5.920 & $2.479-14.139$ & 5.602 & $2.337-13.431^{*}$ \\
Non-excessive total GWG (reference) & 0.448 & $0.248-0.841$ & 0.429 & $0.235-0.783^{*}$ \\
\hline
\end{tabular}

${ }^{*}$ Statistically significant

${ }^{a}$ Adjusted for age

${ }^{\mathrm{b}}$ Adjusted for height, age, and parity

${ }^{c}$ Adjusted for scarred uterus

${ }^{\mathrm{d}}$ Adjusted for age

${ }^{\mathrm{e}}$ Adjusted for scarred uterus. The factors for adjustment are as shown in Tables 1 and 2, which are significant differences or potential differences by Chi-square test

significant association between pre-pregnancy obesity and the severity of preeclampsia, preterm birth, cesarean section, and LGA in patients with GDM complicated by preeclampsia.

Many studies have shown that excessive total GWG increases the risk of preterm birth, C-sect, and LGA [20]. The present study also found that excessive total GWG was a risk factor for LGA in patients with GDM complicated by preeclampsia [21]. However, there are inconsistent reports. It seems difficult to describe accurately with total GWG alone [22]. In 2009, IOM guidelines were proposed for weight control of pregnant women with different BMI, which included total pregnancy and specific gestational periods. The criteria of insufficient, adequate, and excessive weight gain in different gestational periods were determined. On the basis of the guideline, researchers tend to describe the relationship between trimester-specific weight gain and adverse pregnancy outcomes [23].

At least three factors influence the weight change of patients with GDM complicated by preeclampsia during pregnancy in different directions: dyslipidemia from early pregnancy promotes weight gain; plasma volume expansion restriction from early pregnancy inhibits weight gain; increasing blood vessel permeability, decreasing plasma colloid osmotic pressure, and edema aggravation in late pregnancy significantly promote weight gain [12]. Thus, the index of total GWG is not suitable for assessment. Trimester-specific weight gain should be used for evaluation.

Our study shows that in patients with GDM complicated by preeclampsia, the excessive GWG of the late trimester, not that of early or middle trimester, increased the incidence of s-PE. Previous researchers have pointed out that short stature is a risk factor for s-PE [24], and our study is consistent with this. Advanced age and multiparous were also risk factors for s-PE in the present study. After regression analysis adjusted for the influence of short stature and advanced age, the excessive GWG of late trimester was still significantly associated with s-PE. This is the first 
time that this phenomenon has been reported. Some studies reported that predelivery obesity increased the risk of s-PE, but they did not identify whether it was due to excessive GWG [25]. Presumably, compared with patients with m-PE, patients with s-PE suffer more damage to vascular endothelial cells and edema aggravation in the late trimester. These factors have synergistic effects with dyslipidemia and promote weight gain in the late trimester. Therefore, when excessive GWG of late trimester occurs in patients with GDM complicated by preeclampsia, we must be vigilant against s-PE and actively strengthen medical intervention.

The present study also found that the excessive GWG of late trimester was a risk factor for C-sect after adjustment for the impact of the scarred uterus, a result which is consistent with Drehmer et al. [23]. Excessive GWG of late trimester may aggravate the deposition of pelvic soft tissue, leading to decrease in the pelvic area. To overcome the obstruction requires strong contraction and increases the difficulty of vaginal delivery. Excessive GWG of late trimester can also aggravate pelvic inflammatory changes, which is also a risk factor for cesarean section [26].

In this study, the excessive GWG of the middle trimester, not that of the early or late trimester, increased the incidence of LGA. Previous researchers also observed a close relationship between LGA and the excessive GWG of middle trimester [23]. The reasons may be that pregnant women diagnosed with GDM in the middle trimester will receive standard weight management in hospitals, which can effectively control the subsequent weight gain in the late trimester but may not effectively reduce the risk of LGA [27]. The infant metabolism in utero could be compromised by the excessive GWG of the mother. The critical window for the programming of neonatal size at birth is before 28 gestational weeks. Late trimester weight gain can not exert a strong effect on birth weight [28].

No association was found between trimesterspecific excessive weight gain and preterm birth in the present study. There were reports that late excessive GWG in underweight pregnant women and late insufficient GWG in obese pregnant women increased the risk of preterm birth [22]. The number of patients with GDM complicated by preeclampsia included in this study was small, and we did not make further stratified comparisons.

The pathogenesis of GDM complicated by preeclampsia is very complex. Some researchers believe that the onset of preeclampsia is earlier than GDM [29]. However, regarding the effect of pathological changes of GDM complicated by preeclampsia on gestational weight gain, there may be opposite characteristics. The middle trimester weight gain reflects more adverse outcomes closely related to the development of GDM, such as LGA, and the late trimester weight gain is more reflective of the severity of preeclampsia. More evidence is needed to prove the hypothesis.

Obstetric complications have a significant impact on the association between GWG and perinatal outcomes. In some respects, the results of this study are inconsistent with previous reports about pregnant women not suffering the coexistence of GDM and preeclampsia. This discrepancy may be due to the unique pathological mechanism of GDM complicated by preeclampsia. The impairment of GDM and preeclampsia affects each other. The weight gain characteristics of GDM complicated by preeclampsia should be different from those of healthy pregnant women and patients with only one disease (GDM or preeclampsia).

IOM's gestational weight control guideline is a major step forward, but it is based on the general population. It is necessary to develop diversified and targeted guidelines for particular disease phenotypes, such as GDM complicated by preeclampsia.

The weakness of this study included the following: (1) It is a retrospective observational study, not prospective intervention research. (2) It is difficult for a single hospital to collect enough cases, so multicenter cooperation is required to collect more clinical cases and conduct detailed stratified studies. (3) The factors affecting the perinatal outcomes of GDM complicated by preeclampsia may not be limited to those mentioned in the present study. More confounding factors need to be taken into 
account, such as socioeconomic factors, blood sugar control, and medical treatment. (4) To explain why trimester-specific weight gain should be associated with perinatal adverse events, in the future study, we plan to conduct in-depth research by measuring some plasma markers (VEGF, PIGF, IL-6, TNF, etc.).

\section{CONCLUSIONS}

It is essential to research the characteristics of trimester-specific weight gain in GDM complicated by preeclampsia. We found that the middle trimester excessive GWG is a risk factor for LGA, the late excessive GWG is a risk factor for s-PE and C-sect, and the excessive total GWG is a risk factor for LGA and a protective factor for the preterm. Obstetric complications have a significant impact on the association between GWG and perinatal outcomes. This study is helpful for carry out risk monitoring in time, identifying early warning signs, and improving maternal and infant health.

\section{ACKNOWLEDGEMENTS}

We thank all participants of the present study.

Funding. No funding or sponsorship was received for this study or publication of this article. The authors funded the article processing charges. All authors have full access to all of the data in this study and take full responsibility for the integrity of the data and accuracy of the data analysis.

Authorship. All named authors meet the International Committee of Medical Journal Editors (ICMJE) criteria for authorship for this article, take responsibility for the integrity of the work as a whole, and have given their approval for this version to be published.

Author Contributions. Xueqin Zhang performed the design of this study. Yunshan Xiao collected data and wrote/edited the manuscript.
Disclosures. Xueqin Zhang and Yunshan Xiao have nothing to disclose.

Compliance with Ethics Guidelines. All procedures performed in studies involving human participants were in accordance with the ethical standards of the ethics committee of Maternal and Child Healthcare Hospital of Xiamen City (ky-2019-006) and with the 1964 Helsinki Declaration and its later amendments or comparable ethical standards. Informed consent was obtained from all individual participants.

Data Availability. The data sets generated and analyzed during the current study are available from the corresponding author on reasonable request.

Open Access. This article is distributed under the terms of the Creative Commons Attribution-NonCommercial 4.0 International License (http://creativecommons.org/licenses/ by-nc/4.0/), which permits any noncommercial use, distribution, and reproduction in any medium, provided you give appropriate credit to the original author(s) and the source, provide a link to the Creative Commons license, and indicate if changes were made.

\section{REFERENCES}

1. Mirghani Dirar A, Doupis J. Gestational diabetes from A to Z. World J Diabetes. 2017;8(12):489-511. https://doi.org/10.4239/wjd.v8.i12.489.

2. Mosca L, Benjamin EJ, Berra K, et al. Effectivenessbased guidelines for the prevention of cardiovascular disease in women-2011 update: a guideline from the American Heart Association. J Am Coll Cardiol. 2011;57(12):1404-23.

3. Roberts JM, Lain KY. Recent Insights into the pathogenesis of pre-eclampsia. Placenta. 2002;23(5):359-72. https://doi.org/10.1053/plac. 2002.0819 .

4. Jin WY, Lin SL, Hou RL, et al. Associations between maternal lipid profile and pregnancy complicatisons and perinatal outcomes: a population-based study from China. BMC Pregnancy Childbirth. 
2016;16:60. https://doi.org/10.1186/s12884-0160852-9.

5. Schneider S, Freerksen N, Rohrig S, Hoeft B, Maul H. Gestational diabetes and preeclampsia-similar risk factor profiles? Early Hum Dev. 2012;88(3):179-84. https://doi.org/10.1016/j.earlhumdev.2011.08.004.

6. International Association of Diabetes and Pregnancy Study Groups ConsensusPanel, Metzger BE, Gabbe SG, et al. International Association of Diabetes and Pregnancy Study Groups recommendations on the diagnosis and classification of hyperglycemia in pregnancy. Diabetes Care. 2010;33(3):676-82.

7. Wei Y, Yang H, Zhu W, et al. International Association of Diabetes and Pregnancy Study Group criteria is suitable for gestational diabetes mellitus diagnosis: further evidence from China. Chin Med J (Engl). 2014;127(20):3553-6.

8. Eiland E, Nzerue C, Faulkner M. Preeclampsia. J Pregnancy. 2012;2012:586578. https://doi.org/10. $1155 / 2012 / 586578$.

9. Scientific research collaborative group of neonatal development in 15 cities ofChina. Neonatal birth weight for gestational age and percentile in 15 cities of China. Zhonghua Er Ke Za Zh. 1989;27:316 (Chinese).

10. Lao TT, Hui ASY, Sahota DS, Leung TY. Maternal height and risk of hypertensive disorders in pregnancy. J Matern Fetal Neonatal Med. 2017;17:1-6. https://doi.org/10.1080/14767058.2017.1410786.

11. Zhou B, Cooperative Meta-analysis Group of the Working Group on obesity in China. Predictive values of body mass index and waist circumference for risk factors of certain related diseases in Chinese adults-study on optimal cut-off points of body mass index and waist circumference in Chinese adults. Asia Pac J Clin Nutr. 2002;11(Suppl):S685-93.

12. Institute of Medicine and National Research Council. Weight gain during pregnancy: reexamining the guidelines. Washington, DC: The National Academies Press; 2009.

13. Zhu WW, Fan L, Yang HX, et al. Fasting plasma glucose at 24-28 weeks to screen for gestational diabetes mellitus: new environment from China. Diabetes Care. 2013;36:2038-40.

14. Li B, Yang H, Zhang W, et al. Fatty acid-binding protein 4 predicts gestational hypertension and preeclampsia in women with gestational diabetes mellitus. PLoS One. 2018;13(2):e0192347. https:// doi.org/10.1371/journal.pone.0192347.
15. Stella CL, O'Brien JM, Forrester KJ, et al. The coexistence of gestational hypertension and diabetes: influence on pregnancy outcome. Am J Perinatol. 2008;25(6):325-9. https://doi.org/10.1055/s-20081078758.

16. Weissgerber TL, Mudd LM. Preeclampsia and diabetes. Curr Diab Rep. 2015;15(3):9.

17. Odegard RA, Vatten LJ, Nilsen ST, Salvesen KA, Austgulen R. Risk factors and clinical manifestations of pre-eclampsia. BJOG. 2000;107:1410-6.

18. Shin D, Song WO. Prepregnancy body mass index is an independent risk factor for gestational hypertension, gestational diabetes, preterm labor, and small- and large-for-gestational-age infants. J Matern Fetal Neonatal Med. 2015;28(14):1679-86.

19. Kim SS, Mendola P, Zhu Y, Hwang BS, Grantz KL. Spontaneous and indicated preterm delivery risk is increased among overweight and obese women without prepregnancy chronic disease. BJOG. 2017;124(11):1708-16.

20. Goldstein RF, Abell SK, Ranasinha S, et al. Association of gestational weight gain with maternal and infant outcomes: a systematic review and metaanalysis. JAMA. 2017;317(21):2207-25.

21. Huang A, Ji Z, Zhao W, Hu H, Yang Q, Chen D. Rate of gestational weight gain and preterm birth in relation to prepregnancy body mass indices and trimester: a follow-up study in China. Reprod Health. 2016;13(1):93. https://doi.org/10.1186/ s12978-016-0204-2 (Published 2016 Aug 12).

22. Chmitorz A, von Kries R. Rasmussen KM, Nehring I, Ensenauer R. Do trimester-specific cutoffs predict whether women ultimately stay within the Institute of Medicine/National Research Council guidelines for gestational weight gain? Findings of a retrospective cohort study. Am J Clin Nutr. 2012;95(6):1432-7. https://doi.org/10.3945/ajcn. 111.033704.

23. Drehmer M, Duncan BB, Kac G, Schmidt MI. Association of second and third-trimester weight gain in pregnancy with maternal and fetal outcomes. PLoS One. 2013;8(1):e54704.

24. Marić I, Mayo JA, Druzin ML, et al. Maternal height and risk of preeclampsia among race/ethnic groups. Am J Perinatol. 2018. https://doi.org/10.1055/s0038-1675205.

25. Durst JK, Tuuli MG, Stout MJ, Macones GA, Cahill AG. Degree of obesity at delivery and risk of preeclampsia with severe features. Am J Obstet Gynecol. 2016;214(5):651.e1-5. https://doi.org/10. 1016/j.ajog.2015.11.024. 
26. Graham LE, Brunner Huber LR, Thompson ME, Ersek JL. Does amount of weight gain during pregnancy modify the association between obesity and cesarean section delivery? Birth. 2014;41:93-9. https://doi.org/10.1111/birt.12095.

27. Oteng-Ntim E, Varma R, Croker H, Poston L, Doyle P. Lifestyle interventions for overweight and obese pregnant women to improve pregnancy outcome: systematic review and meta-analysis. BMC Med. 2012;10:47. https://doi.org/10.1186/1741-7015-1047 (Published 2012 May 10).
28. Broskey NT, Wang P, Li N, et al. Early pregnancy weight gain exerts the strongest effect on birth weight, posing a critical time to prevent childhood obesity. Obesity. 2017;25:1569-76. https://doi.org/ $10.1002 /$ oby. 21878 .

29. Košir Pogačnik R, Trojner Bregar A, Lučovnik M, et al. The effect of interaction between parity, gestational diabetes, and pregravid obesity on the incidence of preeclampsia. J Matern Fetal Neonatal Med. 2018;19:1-4. https://doi.org/10.1080/ 14767058.2018 .1509311 . 